\title{
Manipulating the disengage operation of covert visual spatial attention
}

\author{
JAMES DANCKERT \\ La Trobe University, Bundoora, Victoria, Australia \\ and \\ PAUL MARUFF \\ Mental Health Research Institute of Victoria, Parkville, Victoria, Australia
}

\begin{abstract}
Processes of covert visual spatial attention have been closely linked to the programming of saccadic eye movements. In particular, it has been hypothesized that the reduction in saccadic latency that occurs in the gap paradigm is due to the prior disengagement of covert visual spatial attention. This explanation has received considerable criticism. No study as yet has attempted to demonstrate a facilitation of the disengagement of attention from a covertly attended object. If such facilitation were possible, it would support the hypothesis that the predisengagement of covert attention is necessary for the generation of express saccades. In two experiments using covert orienting of visual attention tasks (COVAT), with a high probability that targets would appear contralateral to the cued location, we attempted to facilitate the disengagement of covert attention by extinguishing peripheral cues prior to the appearance of targets. We hypothesized that the gap between cue offset and target onset would facilitate disengagement of attention from a covertly attended object. For both experiments, responses to targets appearing after a gap were slower than were responses in the no-gap condition. These results suggest that the prior offset of a covertly attended object does not facilitate the disengagement of attention.
\end{abstract}

The covert orienting of visual attention task (COVAT), described initially by Posner (1980), provides a valid and reliable measure of an individual's ability to direct visuospatial attention to different areas of the visual field without accompanying eye movements. The COVAT requires subjects to keep their eyes fixed on a central location and respond manually to the onset of a target in one of two peripheral locations at either the left or the right of fixation. Before the target appears, a spatial cue is flashed at one of the two peripheral locations. The target can then appear either at the location indicated by the peripheral cue or at the location contralateral to the cue in the opposite visual field. In previous COVAT studies, targets have usually appeared at the cued location with an equal $(50 \%)$ or greater than equal $(80 \%)$ probability. When the time interval between cue and target (stimulus onset asynchrony; SOA) is less than approximately $250 \mathrm{msec}$, target detection, measured using reaction time (RT), is faster when targets appear at the cued location than when targets appear at the location contralateral to the cue (Posner, 1980; Posner \& Cohen, 1984; Posner, Inhoff, \& Friedrich, 1987; Posner \& Petersen, 1990; Rafal \& Henik, 1994). This facilitation of RT occurs because peripheral cues attract the attentional focus automatically and targets are detected faster when they appear at the lo-

Correspondence should be addressed to J. Danckert, c/o P. Maruff, Neurophysiology and Neurovisual Unit, Mental Health Research Institute of Victoria, Parkville, Victoria, Australia 3052 (e-mail: james@) neuro.mhri.edu.au). cation where the cue has prefocused attention (Yantis \& Jonides, 1990). When targets appear in the contralateral location to the cue, subjects need to disengage their attention from the peripheral cue, move the attentional focus to the opposite visual field where the target has actually appeared, and then reengage attention on the target before a response can be made (Posner, 1988). The magnitude of the difference between RT to targets appearing at the cued location and RT to targets appearing contralateral to the cue (e.g., RT to targets contralateral to cue minus RT to targets at cue), or the cue effect size, has been inferred to reflect the additional processing time associated with the need to redirect covert attention following incorrect spatial cues (Bowman, Brown, Kertzman, Schwarz, \& Robinson, 1993; Maruff \& Currie, 1995; Maruff, Pantelis, Danckert, Smith, \& Currie, 1996).

There is now converging evidence to suggest that the cognitive operations involved in the redirection of attention can be inhibited or facilitated independently of each other. For example, COVAT studies show that there is a deficit specific to the disengage operation in patients with unilateral parietal lobe lesions on trials in which cues appear in the contralesional visual field and targets appear in the ipsilesional visual field (Petersen, Robinson, \& Currie, 1989; Posner, 1988; Posner, Walker, Friedrich, \& Rafal, $1984,1987)$. In patients with progressive supranuclear palsy, which is a neurodegenerative disease predominantly involving the midbrain, a specific deficit in the move operation has been reported for the redirection of attention on the vertical but not the horizontal meridian (Rafal, 
Posner, Friedman, Inhoff, \& Bernstein, 1988). A specific deficit in the engage operation has been found in patients with unilateral thalamic lesions when they are required to respond to targets in the contralesional visual field, regardless of the location of the preceding cue (Rafal \& Posner, 1987). Conversely, the time necessary to complete the disengage operation is facilitated in young normal subjects who have been given the catecholiminergic antagonist droperidol (Clark, Geffen, \& Geffen, 1989) and in patients with advanced Parkinson's disease (Clark et al., 1989). Research has also shown that highly skilled athletes show a facilitation in the engage operation of covert visual spatial attention (Nougier, Stein, \& Bonnel, 1991).

Just as the individual operations of covert visual attention can be facilitated or inhibited independently of each other, there is now emerging evidence that the operations of overt visual attention can also be inhibited (Rafal, Calabresi, Brennan, \& Sciolto, 1989; Rafal \& Henik, 1994) and facilitated to some extent (Fischer \& Weber, 1993; Kingstone \& Klein, 1993; Rafal et al., 1989; Tam \& Ono, 1994; Tam \& Stelmach, 1993). In particular, the conditions associated with the facilitation of saccadic eye movements have received significant attention in recent literature. A number of studies have shown that the latency of a saccadic eye movement made from a central fixation point to a sudden-onset target in the periphery of the visual field is approximately $200-250 \mathrm{msec}$ (Fischer \& Weber, 1993; Kingstone \& Klein, 1993; Reuter-Lorenz $\&$ Fendrich, 1992). However, when the offset of the fixation point occurs $150-200 \mathrm{msec}$ before the onset of the target, the mean latency of saccades is reduced significantly. This facilitation of saccadic latency has been termed the "gap effect" (Fischer \& Weber, 1993). The introduction of a temporal gap leads to a bimodal distribution of saccadic latencies with a peak of saccade latencies around 100 msec or less, termed "express saccades," coupled with the peak of regular saccades around 200300 msec (Fischer \& Weber, 1993). Although the existence of these express saccades has been controversial, the gap effect has been shown to be a robust phenomenon (Fischer \& Weber, 1993; Kingstone \& Klein, 1993; Tam \& Ono, 1994; Tam \& Stelmach, 1993).

On the basis of the Posner (1988) model for the cognitive operations involved in the orienting of covert attention, Fischer and co-workers (Braun \& Breitmeyer, 1990; Fischer \& Breitmeyer, 1987; Fischer \& Weber, $1993)$ proposed that the reduction in saccadic latency found in the gap task occurs because the introduction of a temporal gap between the offset of the fixation point and the onset of the target allows the disengagement of covert attention to occur before the target appears. They have hypothesized that it is necessary to disengage covert attention from the point of fixation before a saccade can be executed. Therefore, experimental conditions that allow the prior disengagement of covert attention reduce the time necessary to prepare a saccade and thus decrease their latency (Braun \& Breitmeyer, 1990). Under conditions in which the onset of the target and the offset of the fixation point are simultaneous, all aspects of saccade prepa- ration must occur after the target has appeared (Braun \& Breitmeyer, 1990; Fischer \& Weber, 1993).

For the conventional gap task, the fixation point for the eyes and that for covert attention are the same. Therefore, in order to test the hypothesis that the prior offset of the fixation point facilitates the covert disengagement of attention independently of the ocular motor system, it has been necessary to design experiments in which the point of fixation for the eyes (fixation point) and the location to which attention is directed covertly (attended point) are separated in the visual field. Braun and Breitmeyer (1990) showed that offset of the attended point, separated from a fixation point by $4^{\circ}$ on the horizontal meridian 200-300 msec before the appearance of the target, leads to a significant facilitation of saccadic latency. Tam and Stelmach (1993) also separated the attended point from the fixation point. In separate blocks of trials subjects were required to attend either to the peripheral stimulus or to the fixation stimulus. Saccadic eye movements were facilitated by prior offset of the fixation point and by offset of the attended point, although the facilitation was greater when the fixation point was offset. Manual RTs were facilitated equally by offset of the fixation point and offset of the attended point. Kingstone and Klein (1993) have argued against the hypothesis that predisengagement of covert attention facilitates the preparation of saccades in the gap paradigm, stating that although previous studies have separated the attended point and fixation point in space, they have not ensured that attention was oriented covertly at the attended point. In their study, they separated the attended point and the fixation point in the visual field. For some trials, subjects were required to actively orient attention to the attended point, whereas for other trials the attended point was irrelevant. Offset of the fixation point was associated with a larger reduction in saccadic latency than was offset of the attended point. Importantly, although offset of the attended point was associated with a small reduction in saccadic latency, this occurred when attention was actively oriented to it and when it was irrelevant. Kingstone and Klein hypothesized that the gap effect is due to two separate processes, a response preparation effect and a fixation offset effect. The response preparation effect is conceptualized as a warning effect arising from to the offset of any item in the visual field (e.g., a fixated, attended, or unattended object) and is thought to have equal effects on both the attentional and the ocular motor systems. The fixation offset effect reduces response competition in the ocular system and thereby reduces saccadic latencies. Although these results argue strongly for an ocular motor explanation of the gap effect, the contribution of covert attention to saccadic latencies in the gap paradigm remains a contentious issue (Fischer \& Weber, 1993; Kingstone \& Klein, 1993; Tam \& Stelmach, 1993).

One alternative approach to investigating the contribution of the covert attentional disengagement to the gap effect has been to have subjects maintain fixation at a central point but to shift their attention covertly to a peripheral location and perform a shape discrimination task. 
Using this paradigm, Mackeben and Nakayama (1993) found that offset of the fixation point $200 \mathrm{msec}$ before the onset of the shape better facilitated decisions of shape orientation than a condition in which the offset of the fixation point and onset of the peripheral shape were simultaneous. This result suggests that prior offset of the fixation point may affect both ocular motor and attentional systems. However, it appears that the extent to which either system is involved may depend on the particular demands of the task used. Although the major debate regarding the gap effect has centered on whether it is the prior disengagement of covert attention that is responsible for the reduction in saccadic latency, no study has sought to investigate the gap effect within the original Posner (1980) paradigm despite the requirement that the attended point and the fixation point are spatially separate in the COVAT paradigm.

In the COVAT with peripheral cues, in which targets appear at the cued location with an equal $(50 \%)$ or greater than equal (e.g., $70 \%-80 \%$ ) probability, the subject's attentional focus is drawn automatically by the cue (Yantis \& Jonides, 1990). Under these conditions at short SOAs, the attentional focus remains at the cued location until the target appears. Therefore, when the target appears opposite the cued location, subjects only begin the disengage, move, and engage operations at the appearance of the target. Recently we reported that subjects began the cognitive operations associated with the redirection of attention away from peripheral cues before the target appeared when there was an $80 \%$ probability that the target would appear in the location contralateral to the cue (Maruff \& Currie, 1995). Under these conditions, RTs to targets appearing contralateral to the cue were significantly faster than RTs to targets appearing at the cued location. As the peripheral cue initiated the reflexive orienting mode of attention, subjects could not avoid orienting to it (Yantis \& Jonides, 1990). Prior expectation that targets would appear opposite the cue, however, allows subjects to disengage attention from the cue and redirect it to the opposite visual hemifield before the target appears (Maruff \& Currie, 1995; Posner, Cohen, \& Rafal, 1982; Rafal \& Henik, 1994). It is clear from these studies that manipulation of the target at cue probability contingencies has affected the way in which covert attention has been disengaged from the cue.

\section{EXPERIMENT 1}

In a conventional COVAT, a peripheral cue remains present until the subject responds to the target. However, a temporal gap between offset of the cue and the onset of the target can be introduced by extinguishing the cue shortly after its onset. It may be the case that a short cue duration will also affect the way in which covert attention can be disengaged and redirected. In the experiments reported here, we used the COVAT with peripheral cues and an $80 \%$ probability that the target would appear in the contralateral location to the cue to investigate whether it was possible to facilitate the disengagement from peripheral cues by presenting the covertly attended object (the peripheral cue) for a very brief time interval prior to target onset. As the fixation point and the attended point were separated in space, we hypothesized that the disengagement of covert attention would be better facilitated when the attended object was presented for a brief time interval $(75 \mathrm{msec})$ than when the attended object remained present for the entire length of the SOA.

\section{Method}

Subjects. Ten subjects, 5 male and 5 female university students, were recruited to participate. The average age was 20.7 years, with a range of 17 to 29 years. All subjects had normal or corrected-tonormal vision.

Apparatus. The COVAT was presented on a computer monitor located $95 \mathrm{~cm}$ from the subject and controlled by an IBM-compatible computer with 1-msec timing for control of stimulus displaying and recording of RT. A central red fixation point subtending $0.2^{\circ}$ of visual angle remained visible throughout an experimental session. The background luminance of the monitor was $8.9 \mathrm{~cd} / \mathrm{m}^{2}$, and the fixation point was presented at a luminance of $35 \mathrm{~cd} / \mathrm{m}^{2}$. The target for the task was a $0.2^{\circ}$ red spot that appeared in either the right or left visual field on the horizontal meridian $8^{\circ}$ from the fixation point. The target was presented at a luminance of $63.2 \mathrm{~cd} / \mathrm{m}^{2}$. Spatial cues for the appearance of a target consisted of a circle subtending $2^{\circ}$ of visual angle, the center of which was $8^{\circ}$ to the left or right of the fixation point on the horizontal meridian. Spatial cues were presented at a luminance of $35 \mathrm{~cd} / \mathrm{m}^{2}$. The target could appear either at the same location as the spatial cue (target at cue; TAC) or at the mirror-symmetric location in the contralateral visual field (target contralateral to cue; TCC). Two versions of the COVAT were given. For each version, there was an $80 \%$ probability that targets would appear in the contralateral location to the cue (TCC). For both versions, the SOA was pseudorandomized among 150, 350, and $550 \mathrm{msec}$. For the no-gap condition, peripheral cues remained visible throughout the length of the SOA and were extinguished only when the subject responded to the target. For the gap condition, peripheral cues remained visible for $75 \mathrm{msec}$ regardless of the SOA. Therefore, the temporal gap between offset of the attended object and onset of the target depended on the length of the SOA. For $150-\mathrm{msec}$ SOA, the gap was $75 \mathrm{msec}$; for the $350-\mathrm{msec} \mathrm{SOA}$, the gap was $275 \mathrm{msec}$; and for the 550 -msec SOA, the gap was $475 \mathrm{msec}$. Each version of the COVAT consisted of 318 trials, of which 300 were spatially cued and 18 were catch trials in which no target followed a cue. Of the spatially cued trials, the TCC condition occurred for 240 trials and the TAC condition occurred for 60 trials. Targets could appear in the left or right visual fields with equal probability. In a single testing session, subjects performed the two versions of the COVAT. The two versions of the COVAT were administered in random order and subjects were given short rest breaks after every 100 trials. For each subject, mean RTs were calculated for each cue type (TAC, TCC) at each SOA (150, 350, and $550 \mathrm{msec}$ ) under the gap and no-gap conditions. These were used to calculate a group mean, which was then subjected to a repeated measures analysis of variance (ANOVA). See Figure 1 for a schematic representation of the COVAT display.

Procedure. Subjects were seated $95 \mathrm{~cm}$ from the screen in a darkened, sound-attenuated room. Subjects were instructed to maintain visual fixation on the central cross in the display and to respond to the presence of a target with a buttonpress. Since previous studies have found that normal subjects have no difficulty maintaining central visual fixation while responding to targets appearing in the pe- 


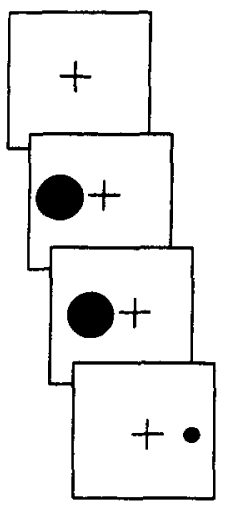

no gap

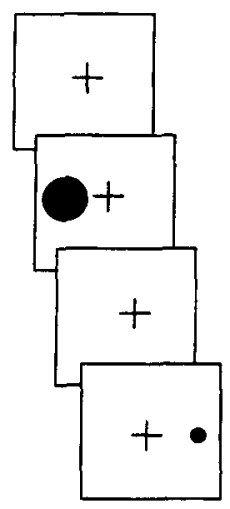

gap

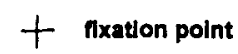

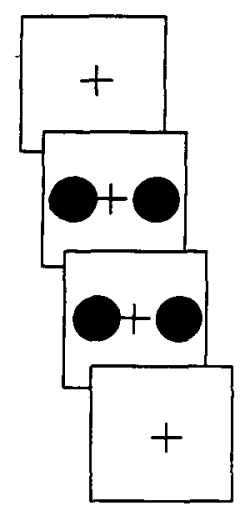

catch trial cue

- targot

Figure 1. Schematic representation of the sequence of screens presented in the covert orienting of visual attention tasks for nogap, gap, and catch trials. Note that in all examples (except the catch trials), the target appears contralateral to the cued location.

riphery, eye movements were not monitored (Maruff \& Currie, 1995; Posner et al., 1982). Subjects were given no prior information concerning the different experimental manipulations.

\section{Results}

RTs of less than 100 and greater than $1,000 \mathrm{msec}$ were deemed to be anticipatory responses and omissions, respectively, and were excluded from further analysis. Less than $3 \%$ of RTs were categorized as anticipatory responses and omissions. For each subject, median RTs were calculated for each cue type at each SOA under both conditions. These medians were submitted to an ANOVA, with gap condition, cue type, and SOA as within-subjects factors.
The ANOVA showed a significant main effect of SOA $[F(2,18)=11.21, p<.01]$ and a significant gap condition $\times$ cue type interaction $[F(1,9)=8.58, p<.05]$. No other main effects or interactions were found to be significant. The mean effects for this experiment can be seen in Figure 2.

Newman-Keuls post hoc tests $(p<.05)$ showed a significant difference between the 150- and 350-msec SOAs but not between the 350- and 550-msec SOAs. The interaction was investigated via one-way ANOVAs, which indicated a significant main effect for both gap and no-gap conditions on RTs to TAC $[F(1,99)=61.15, p<.001]$ as well as on RTs to TCC $[F(1,99)=21, p<.001]$. Inspection of group means showed that RTs to TAC were slower in the gap condition $(\mu=329.4)$ than were RTs to TAC in the no-gap condition $(\mu=261.65)$. This pattern of RTs was the same for RTs to TCC $(\mu=278.2$ in the gap condition compared with $\mu=238.5$ in the no-gap condition); however, the difference between conditions was greater for RTs to TAC $(67.67 \mathrm{msec})$ than for RTs to TCC $(39.7 \mathrm{msec})$. Figure 3 is a graph of this interaction.

\section{Discussion}

The results of this experiment indicate that a temporal gap between the offset of the peripheral cue and the onset of a target does not facilitate the disengagement of covert attention. Since overt ocular motor processes were not manipulated in this experiment, the results suggest that the disengagement of covert attention, in the context of the Posner (1988) model, is neither a necessary nor a sufficient condition for the ocular motor gap effect. Therefore, the current results are consistent with those of previous research suggesting that the reduction in saccadic latency found on the ocular motor gap task is related to ocular motor processes rather than to processes associated with covert visual attention (Kingstone \& Klein, 1993). In the

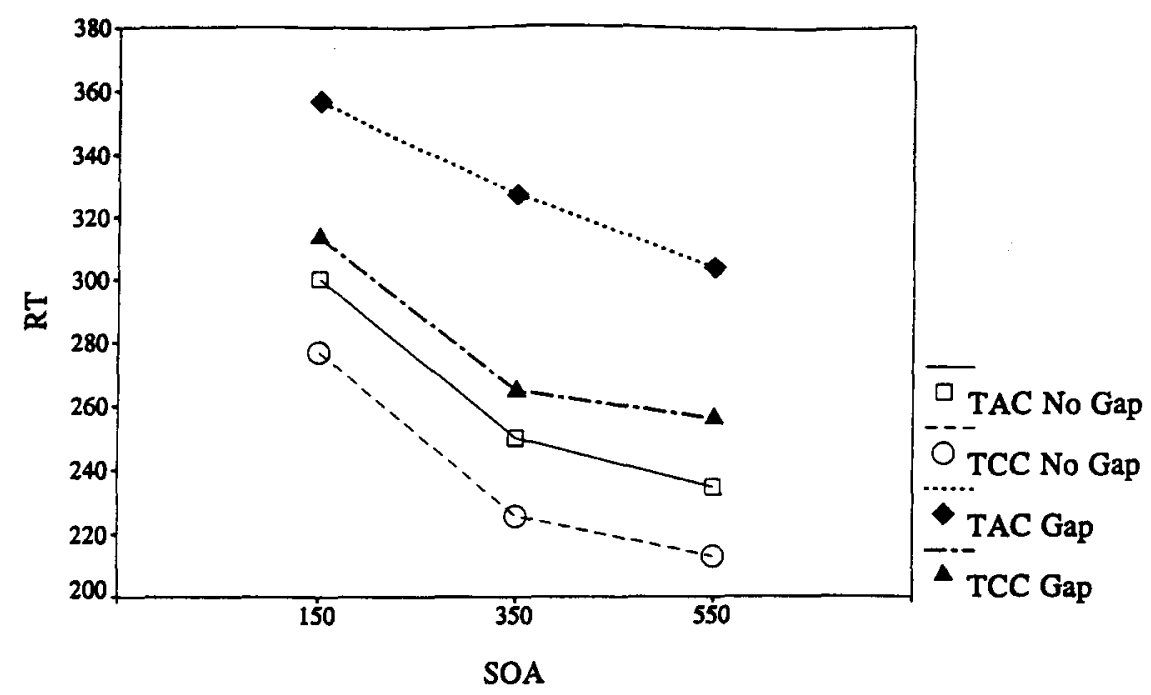

Figure 2. Group mean reaction times (RTs) for both no-gap and gap conditions. TAC, target at cue; TCC, target contralateral to cue; SOA, stimulus onset asynchrony. Note that both conditions were $20 \%$ target at cue probability ratios. 


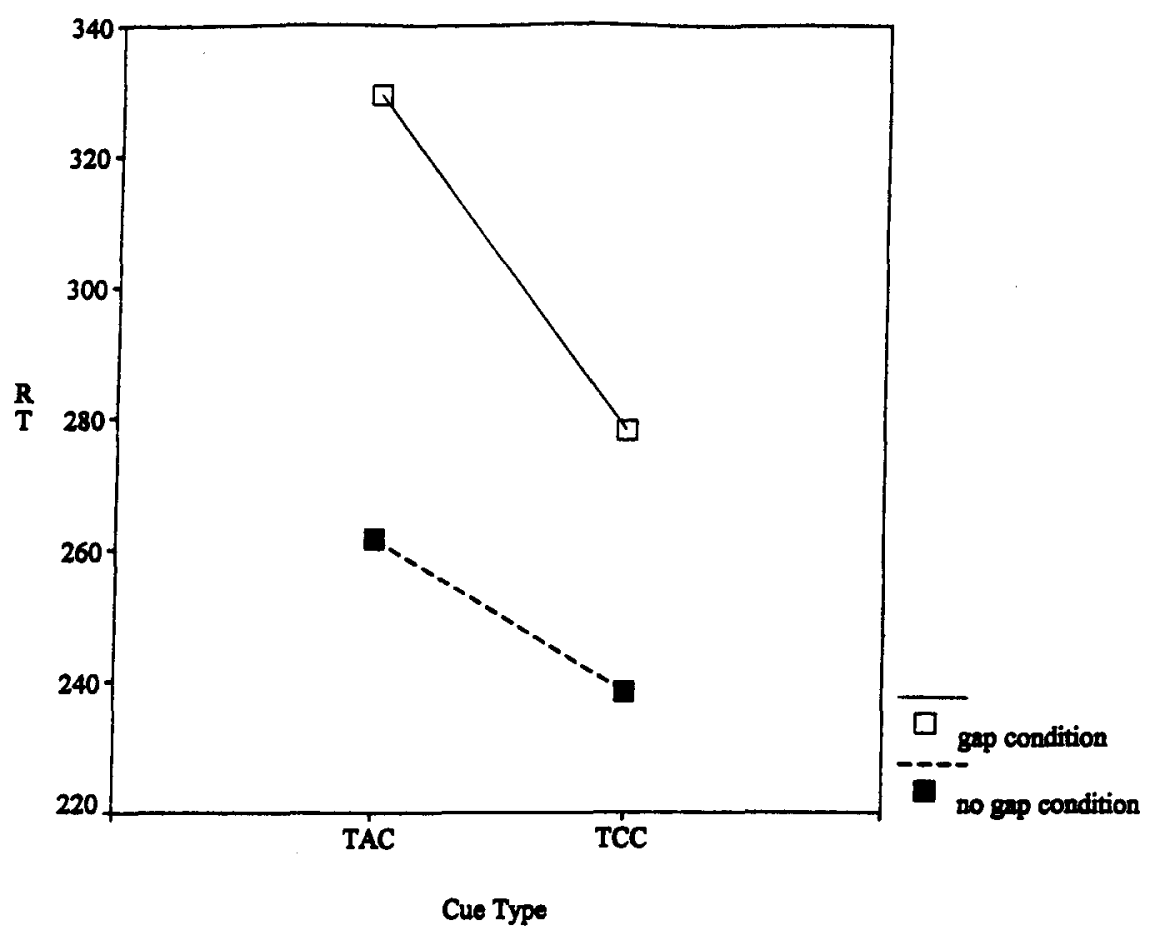

Figure 3. Interaction effect $\times$ cue types. TAC, target at cue; TCC, target contralateral to cue.

present experiment, RTs were faster to TCC trials than to TAC trials in both conditions, indicating that subjects were able to use the probability information contained in spatial cues to redirect their attention away from the peripheral cue before the target appeared. Since subjects' RTs were faster for TCC trials, they must have begun to disengage attention from the cue before the target appeared. We hypothesized that the very brief presentation of the cue would allow the disengagement of covert attention from the cue to occur more rapidly than when the cue remained visible until the target appeared. However, contrary to our expectations, RTs to TCC and TAC trials were increased when the cue was extinguished before the target appeared. These results suggest that when attentional shifts are covert, the very brief presentation of spatial cues slows the disengagement of attention. Previous studies have shown that the presence of visual landmarks improves performance on the COVAT (Bowman et al., 1993; Mangun, Hansen, \& Hillyard, 1987). In the no-gap condition of this experiment, the peripheral cues remained present for the entire SOA, thereby providing a visual landmark at the site of the cue. When subjects were orienting attention away from the cue (i.e., TCC) to a blank visual hemifield, the presence of the cue in the opposite visual hemifield may have provided some point of reference for orienting covert attention. It may have been the case that in the gap condition, the absence of a visual landmark in the display (i.e., the short cue duration meant that the display was blank except for the central fixation point) was responsible for the generalized slowing of RTs.
In the present experiment, the RT slowing associated with the gap condition was greater on TAC trials (Figure 3). The high probability of TCC trials led subjects to direct their attention away from peripheral cues to the opposite visual field. For TAC trials, the appearance of the target would require subjects to make a second shift of attention back to the location where the cue had been presented initially. Since this second shift of attention was initiated by the appearance of the target and not the cue, the length of time for which the spatial cue was presented should have had no effect on RT to TAC. Therefore, if the gap condition affected only the disengagement of covert attention from the initial cue, then RT to TAC and TCC trials should have been slowed to the same extent. The effect of the presence of the cue in the no-gap condition discussed above may also provide an explanation of the interaction effect. In the no-gap condition, a peripheral cue remained visible throughout the entire SOA. Previous studies have shown that the presence of visual landmarks improves performance on the COVAT (Bowman et al., 1993; Mangun et al., 1987). In the nogap condition, the peripheral cue would provide a visual landmark for the cued location only. Therefore, RTs to TAC trials may have been improved because of the presence of the visual landmark, whereas no such improvement would have occurred in the gap condition because shifts of attention back to the target were made without any such landmark. Thus, the absence of visual landmarks may have been associated with the additional slowing on TAC trials in the gap condition. 


\section{EXPERIMENT 2}

The aim of Experiment 2 was to investigate the effect that visual landmarks presented around the possible target locations would have on the pattern of RTs to TAC and TCC trials in the gap and no-gap conditions. With visual landmarks, the initial attentional shifts away from cues, on both TCC and TAC trials, as well as the targetinitiated shifts back to the cued locations on TAC trials, would be performed under the same stimulus conditions. We hypothesized that the presence of visual landmarks would provide the necessary conditions under which a gap effect for disengaging covert attention could be observed. That is, the presence of visual landmarks at the site of both possible target locations in the gap condition would facilitate the disengagement of attention, producing faster RTs to TCC in the gap condition than in the no-gap condition.

\section{Method}

Subjects. Four subjects recruited from the Mental Health Research Institute of Victoria, 3 male and 1 female, participated in this experiment. The average age was 26.5 years, with a range of 21 to 35 years.

Apparatus. The experimental apparatus was identical to that described in Experiment 1. The stimulus display was changed so that for both the gap and no-gap conditions, visual landmarks subtending $2^{\circ}$ of visual angle whose centers were located $8^{\circ}$ to the right and left of fixation on the horizontal meridian remained visible throughout an entire testing session. The luminance of visual landmarks was $35 \mathrm{~cd} / \mathrm{m}^{2}$. Peripheral spatial cues now consisted of luminance doubling of a landmark.

Design and Procedure. The two versions of the COVAT used in Experiment 2 were identical to those used in Experiment 1 with the exception that visual landmarks had now been added. The administration and data analysis were also identical to those in Experiment I. Figure 4 schematically represents the stimulus displays used in Experiment 2.

\section{Results}

Omissions and anticipatory responses were treated in the same way as in Experiment 1, and once again there were very few omissions and these received no further analysis. A paired-samples $t$ test on the anticipatory RTs showed no significant difference between the two conditions $[t(8)=-.84, p>.05]$. The percentage of anticipatory responses was less than $3 \%$ for each condition.

An ANOVA showed significant main effects on RT for condition $[F(1,33)=13.87, p<.01]$, SOA $[F(2,33)=$ $19.4, p<.01]$, and cue type $[F(1,33)=48.11, p<.01]$. No interactions were found to be significant. Mean effects of this experiment can be seen in Figure 5.

Newman-Keuls post hoc tests $(p<.05)$ showed a significant difference between the 150- and 350-msec SOAs but not between the 350- and 500-msec SOAs. Inspection of group means indicated that in both conditions RTs to TCC were faster than RTs to TAC ( $\mu=240.125$ for TCC; $\mu=286.188$ for TAC). In addition, mean RTs for TAC and TCC trials were significantly faster in the nogap condition $(\mu=250.792)$ than in the gap condition $(\mu=275.52)$.
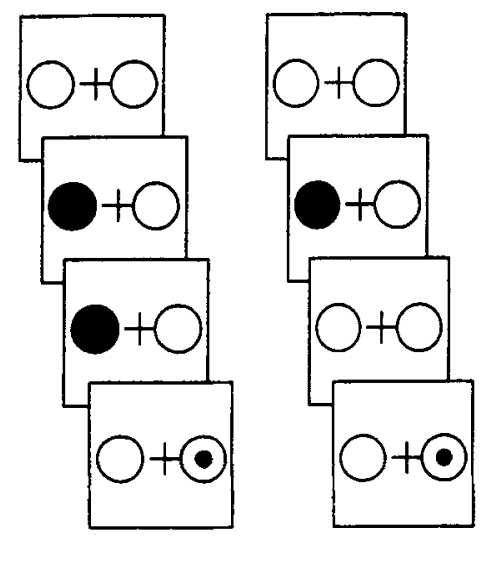

No Gap

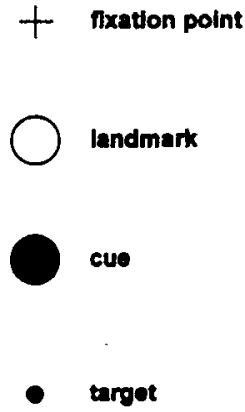

Figure 4. Schematic representation of the sequence of screens for the covert orienting of visual attention tasks used in Experiment 2. Note that the display is identical to that used in Experiment 1 (see Figure 1) with the exception of the addition of visual landmarks either side of the visual fixation point.

\section{Discussion}

The results of Experiment 2 once again show that RTs to both TAC and TCC trials were slower in the gap condition than in the no-gap condition. However, with visual landmarks, RTs to TAC and TCC trials were slowed to the same extent in the gap condition. This suggests that in Experiment 1, the additional slowing found for TAC trials in the gap condition occurred because there was no landmark for target-initiated shifts of attention back to the cued location. The magnitude of the RT difference between TCC and TAC trials was equal in the gap and nogap conditions in Experiment 2 and in the no-gap condition in Experiment 1 . Thus, when the peripheral cue was visible throughout the SOA (Experiment 1, no-gap condition) or when visual landmarks were presented on all trials (Experiment 2, both conditions), RTs to TAC trials were improved more than when no landmark was visible (Experiment 1, gap condition). As in Experiment 1, RTs to TCC trials were faster than RTs to TAC trials, indicating that for TCC trials, subjects had begun the disengagement of attention before the target appeared. However, the slower RTs for both TAC and TCC trials in the gap condition indicated that, even with visual landmarks, the offset of the peripheral cue did not facilitate the disengagement of covert attention.

\section{GENERAL DISCUSSION}

The results of Experiments 1 and 2 suggest that for covert attentional shifts measured using the COVAT, the offset of the attended point does not facilitate the disengagement of attention. In Experiments 1 and 2, there was a high probability that targets would appear in the location contralateral to the cue. As a result, RTs to targets that appeared contralateral to the cued location were faster than RTs to targets at the cued location. This fa- 


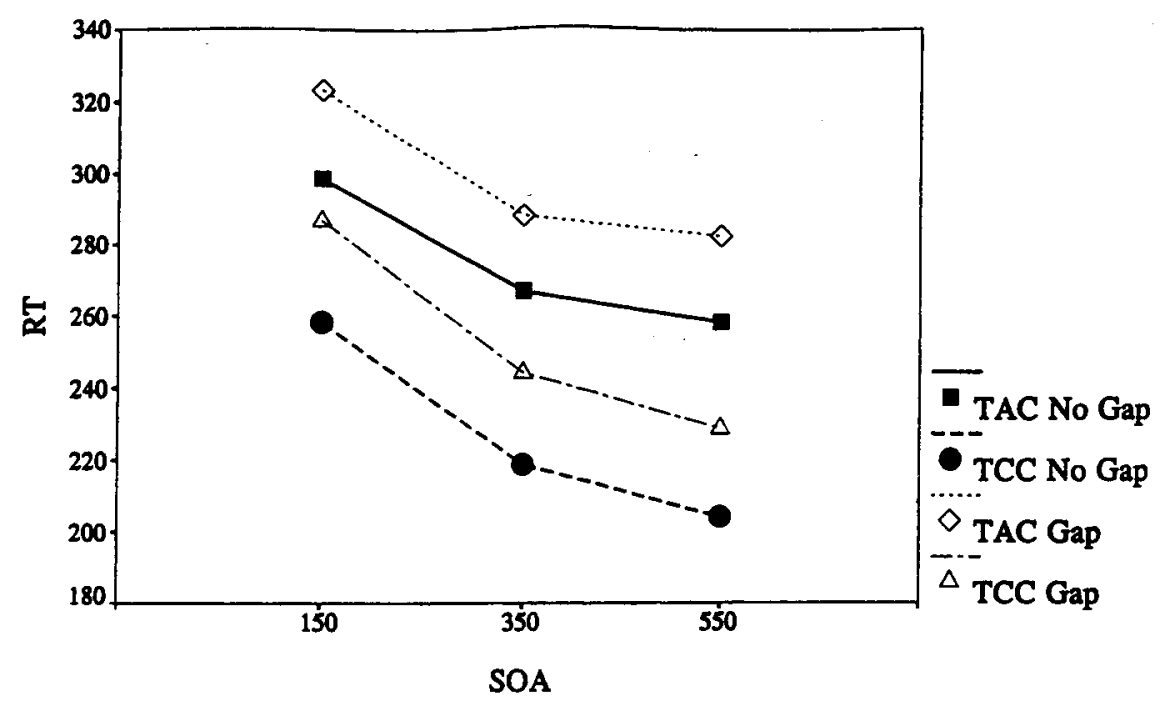

Figure 5. Group mean reaction times (RTs) for both the no-gap and gap conditions with the addition of visual landmarks either side of the fixation point. TAC, target at cue; TCC, target contralateral to cue; SOA, stimulus onset asynchrony.

cilitation of RTs suggests that although the peripheral luminance increase attracted the attentional focus to the peripheral cue, subjects disengaged and moved their attention from the cue to the contralateral location before the target appeared. The slower RTs for targets appearing at the cued location indicates that for these trials, subjects had to complete a second shift of attention, initiated by the appearance of the target, back to the cued location. In both experiments, we attempted to facilitate the disengagement of attention from the peripheral cue by presenting the cue for a very brief time period. On the basis of findings from studies using ocular motor gap tasks, we hypothesized that covert attention would be disengaged more rapidly when there was no attended object present in the visual field.

Previous studies have shown that peripheral luminance increases automatically attract an attentional focus (Theeuwes, 1991; Yantis \& Jonides, 1990 ). On the basis of the probability information, also contained in peripheral cues, subjects would expect that the target would appear in the location contralateral to the cue and would begin to disengage attention from the cue immediately (Maruff \& Currie, 1995). When the peripheral cue remained present throughout the SOA, subjects would have to disengage attention from a visible object. When the peripheral cue was very brief, the object would disappear and subjects would be disengaging their attention from a location in a blank visual hemifield. The absence of any facilitation of RT in the gap condition suggests that the rapid offset of the attended cue did not facilitate the disengagement of attention from the peripheral cue. Thus the present results do not support the hypothesis offered by Fischer and Weber (1993) that offset of the attended point allows the rapid disengagement of covert attention in the ocular motor gap task. For the ocular motor gap task, the offset of the fixation point is more likely to be related to disengagement of the ocular motor fixation, as proposed by Kingstone and Klein (1993).

The cognitive operations necessary to detect a target appearing opposite the cued location are hypothesized to be the disengagement of attention from the cue, the movement of attention to the opposite location, and the reengagement of attention at the target site (Maruff \& Currie, 1995; Posner, 1988; Rafal \& Henik, 1994). In both experiments, subjects were able to show a significant RT advantage for TCC, suggesting that the ability to disengage attention from the cue was unaffected by shorter cue times. Had shorter cue times created a deficit in the ability to disengage attention from the cue, an RT pattern showing an advantage for TAC or at least no advantage for TCC over TAC would have been expected. This was not the case, suggesting that the disengage operation of covert visual spatial attention was unaffected by the shorter cue times of the gap condition.

The advantage for RTs to TCC in both conditions was evident at the first SOA, indicating that even at the shortest SOA, subjects had enough time to move their attention to the opposite location prior to the appearance of the target. If the shorter cue time of the gap condition had differentially affected subjects' ability to move covert attention, the RT advantage for TCC might have become evident only at longer SOAs. This was not the case, suggesting that short cue times had not affected the move operation of covert visual spatial attention.

The introduction of a gap between cue offset and target onset may have affected subjects' ability to engage targets once they appeared. Similar RT patterns have been observed in patients with unilateral thalamic lesions - that is, in an area of the brain hypothesized to be responsible for the covert engagement of attention (Petersen, Robinson, \& Morris, 1987; Rafal \& Posner, 1987). The difficulty subjects had in engaging targets appearing at both loca- 
tions in the gap task was evident even when visual landmarks were present at the site of the cue and target, suggesting that the temporal gap between cue offset and target onset, rather than the stimulus display, was responsible for the slower RTs. Several studies have indicated that both human and animal performance of covert attentional tasks is improved by the presence of visual landmarks (Bowman et al., 1993; Mangun et al., 1987); however, the presence of landmarks in this study had no effect on the operations of covert visual spatial attention.

The interpretation of the effect of the gap condition offered above suggests a specific difficulty in engaging targets whether they appeared at the cued location or opposite that location, and regardless of the presence or absence of visual landmarks in the stimulus display. A second plausible explanation can be offered. We have already argued that to show an RT advantage for TCC when the cues used are peripheral luminance increases, it is necessary for an endogenous orienting mode of attention to redirect attention away from the cue following the exogenous orienting response toward the cue (Maruff \& Currie, 1995; Maruff et al., 1996; Rafal \& Henik, 1994; Yantis \& Jonides, 1990). Given that an RT advantage for TCC was evident for both conditions of each experiment, and that the slower RT pattern in the gap conditions was systematic, it may be unreasonable to assume that one specific operation of covert attention was affected by the temporal gap. Instead, it may be the case that the shorter cue time of the gap conditions caused a generalized slowing of the endogenous mode of orienting affecting all operations of covert attention equally. This is a post hoc hypothesis and would need to be investigated by further research. Several studies have shown, however, that by manipulating the type of peripheral cue used (i.e., manipulating cue time, luminance, size, etc.), RT patterns showing facilitation for one target type over another can also be manipulated. The RT patterns in this study were unaffected by the short cue times or the presence or absence of landmarks; however, it may have been the case that the speed with which the cognitive operations of covert orienting were employed was affected by these changes in the stimulus display.

In this study, we have been unable to facilitate the disengagement of covert attention from a covertly attended object when ocular motor systems remained centrally fixated. Previous studies that have shown facilitation of covert attentional operations have relied on the offset of the central fixation point for facilitation to occur (e.g., Mackeben \& Nakayama, 1993; Tam \& Stelmach, 1993). It may be that for facilitation of covert mechanisms to occur, overt mechanisms must also be in a disengaged state. This would lend support to Kingstone and Klein's (1993) interpretation of the gap effect as a fixation offset effect. In the present study, the ocular motor system remained engaged throughout an entire trial sequence. Regardless of whether an eye movement is to be made or not, it may be necessary for the ocular motor system to be in a disengaged state for facilitation of covert mech- anisms to occur. Cells in the superior colliculus have been shown to fire during ocular fixation (Muñoz \& Wurtz, 1993). The superior colliculus has also been hypothesized to be the neural substrate underlying the covert movement of attention (Posner, 1988; Posner \& Dehaene, 1994). It may be necessary for overt mechanisms to be in a disengaged state (i.e., for the fixation cells of the superior colliculus to be released) for any facilitation of covert mechanisms to become evident. Further neurophysiological as well as neurobehavioral research will be necessary to investigate this hypothesis.

\section{REFERENCES}

Bowman, E. M., Brown, V. J., Kertzman, C., Schwarz, U., \& RobinSON, D. L. (1993). Covert orienting in Macaques I. Effects of behavioural context. Journal of Neurophysiology, 70, 431-443.

Braun, D., \& Breitmeyer, B. (1990). Effects of reappearance of fixated and attended stimuli upon saccadic reaction time. Experimental Brain Research, 81, 318-324.

Clark, C. R., Geffen, G. M., \& Geffen, L. B. (1989). Catecholamines and the covert orientation of attention in humans. Neuropsychologia, 27, 131-139

Fischer, B., \& Breitmeyer, B. (1987). Mechanisms of visual attention revealed by saccadic eye movements. Neuropsychologia, 25, 73-83.

Fischer, B., \& WEBER, H. (1993). Express saccades and visual attention. Behavioural \& Brain Sciences, 16, 553-610.

Kingstone, A., \& KLein, R. M. (1993). Visual offsets facilitate saccadic latency: Does predisengagement of visuospatial attention mediate this gap effect? Journal of Experimental Psychology: Human Perception \& Performance, 19, $1251-1265$.

MaCkeben, M., \& NaKayama, K. (1993). Express attentional shifts Vision Research, 33, 85-90.

Mangun, G. R., Hansen, J. C., \& Hillyard, S. A. (1987). The spatial orienting of attention: Sensory facilitation or response bias? In R. Johnson, Jr., J. W. Rohrbaugh, \& R. Parasuraman (Eds.), Current trends in event related potential research (pp. 118-124). New York: Elsevier.

MARUFF, P., \& CURRIE, J. (1995). An attentional grasp reflex in patients with Alzheimer's disease. Neuropsychologia, 33, 689-701.

Maruff, P., Pantelis, C., Danckert, J., Smith, D., \& Currie, J. (1996). Deficits in the endogenous redirection of covert visual attention in chronic schizophrenia. Neuropsychologia, 34, 1079-1084.

MuÑoz, D. P., \& Wurtz, R. H. (1993). Fixation cells in monkey superior colliculus I. Characteristics of cell discharge. Journal of Neurophysiology, 70, 559-575

Nougier, V., Stein, J. F., \& Bonnel, A.-M. (1991). Information processing in sport and orienting of attention. International Journal of Sport Psychology, 22, 307-327.

Petersen, S. E., Robinson, D. L., \& Currie, J. N. (1989). Influences of lesions of parietal cortex on visual spatial attention in humans. $E x$ perimental Brain Research, 76, 267-280.

Petersen, S. E., Robinson, D. L.; \& Morris, D. J. (1987). Contributions of the pulvinar to visual spatial attention. Neuropsychologia, 25, 97-195.

PosNer, M. I. (1980). Orienting of attention. Quarterly Journal of Experimental Psychology, 32, 3-25.

POSNER, M. I. (1988). Structures and functions of selective attention. In T. Boll \& B. Bryant (Eds.), Clinical neuropsychology and brain function: Research, measurement, and practice (pp. 169-202). Washington, DC: American Psychological Association.

Posner, M. I., \& Cohen, Y. (1984). Components of visual orienting. In H. Bumpy \& T. U. Bouwhuis (Eds.), Attention and performance $X$. London: Erlbaum.

Posner, M. I., Cohen, Y., \& Rafal, R. D. (1982). Neural systems control of spatial orienting. Philosophical Transactions of the Roval Society of London: Series $B, 48,318-337$

Posner, M. I., \& Dehaene, S. (1994). Attentional networks. Trends in Neurosciences, 17, 75-79. 
Posner, M. I., INHOFF, A. W., \& Friedrich, F. J. (1987). Isolating attentional systems: A cognitive-anatomical analysis. Psychobiology, 15, 107-121.

Posner, M. I., \& Petersen, S. E. (1990). The attention system of the human brain. Annual Review of Neuroscience, 13, 25-42.

Posner, M. I., Walker, J. A., Friedrich, F. J., \& Rafal, R. D. (1984). Effects of parietal injury on covert orienting of attention. Journal of Neuroscience, 4, 1863-1874.

Posner, M. I., Walker, J. A., Friedrich, F. J., \& Rafal, R. D. (1987), How do the parietal lobes direct covert attention? Neuropsychologia, 25, 135-145.

Rafal, R. D., Calabresi, P. A., Brennan, C. W., \& Sciolto, T. K. (1989). Saccade preparation inhibits reorienting to recently attended locations. Journal of Experimental Psychology: Human Perception \& Performance, 15, 673-685.

RAFAL, R. D., \& HeNIK, A. (1994). The neurology of inhibition. Integrating controlled and automatic processes. In D. L. Dagenbach \& V. Carr (Eds.), Inhibitory processes in attention, memory and language (pp. 1-50). New York: Academic Press.

RAFAL, R. D., \& PosNer, M. I. (1987). Deficits in visual spatial attention following thalamic lesions. Proceedings of the National Academy of Sciences, 84, 7349-7353.
Rafal, R. D., Posner, M. I., Friedman, J. H., Inhoff, A. W., \& BernSTEIN, E. (1988). Orienting of visual attention in supranuclear palsy. Brain, 111, 267-280.

REUTER-LoRENZ, P. A., \& FENDRICH, R. (1992). Oculomotor readiness and covert orienting: Differences between central and peripheral cues. Perception \& Psychophysics, 52, 336-344.

TAM, W. J., \& ONO, H. (1994). Fixation disengagement and eye-movement latency. Perception \& Psychophysics, 56, 251-260.

TAM, W. J., \& Stelmach, L. B. (1993). Viewing behavior: Ocular and attentional disengagement. Perception \& Psychophysics, 54, 211222.

THEEUWES, J. (1991). Exogenous and endogenous control of attention: The effect of visual onsets and offsets. Perception \& Psychophysics, 49, 83-91.

YANTIS, S., \& JONIDES, J. (1990). Abrupt visual onsets and selective attention: Voluntary versus automatic allocation. Journal of Experimental Psychology: Human Perception \& Performance, 16, 121-134.

(Manuscript received March 24, 1995; revision accepted for publication May 20, 1996.) 